\title{
BOUNDEDNESS OF CLASSICAL CALDERÓN-ZYGMUND CONVOLUTION OPERATORS ON PRODUCT HARDY SPACE
}

\author{
ChaOdiang TAN
}

\begin{abstract}
It is well known that the classical Calderón-Zygmund convolution operators are bounded on the classical Hardy spaces. In this paper, we prove that these operators are also bounded on the product Hardy spaces.
\end{abstract}

\section{Introduction}

The extensive study of the classical Hardy spaces $H^{p}\left(\mathbb{R}^{n}\right)$ began with the fundamental works of Stein-Weiss [17] and Fefferman-Stein [6]. These spaces played an important role in harmonic analysis. Especially, as an extension of the $L^{p}(p>1)$ boundedness, the classical Calderón-Zygmund operators are bounded on the classical Hardy spaces $H^{p}\left(\mathbb{R}^{n}\right)$ and from $H^{p}\left(\mathbb{R}^{n}\right)$ to $L^{p}\left(\mathbb{R}^{n}\right)$ for $0<p \leq 1$.

The product Hardy space was introduced by Gundy and Stein [9]. However, the product Hardy space is essentially different from the classical Hardy space. For instance; see [1-3]. Nevertheless, the product singular integrals began with convolution operators studied by R. Fefferman and Stein [8] and continued with non convolution operators introduced by Journé [13], are bounded on the product Hardy space $H^{p}\left(\mathbb{R}^{n} \times \mathbb{R}^{m}\right)$ and from $H^{p}\left(\mathbb{R}^{n} \times \mathbb{R}^{m}\right)$ to $L^{p}\left(\mathbb{R}^{n+m}\right)$; see $[4,10]$. It is not difficult to see that the product Calderón-Zygmund convolution operators in general need not to be bounded on the classical Hardy spaces. Indeed, let $T$ be the double Hilbert transform on $\mathbb{R}^{2}$, i.e., $T(f)=p . v \cdot \frac{1}{x_{1} x_{2}} * f$. Set $a\left(x_{1}, x_{2}\right)=\chi_{\left(0 \leq x_{1} \leq 1\right)}\left(x_{1}\right)\left[\chi_{\left(0 \leq x_{2} \leq 1\right)}\left(x_{2}\right)-\right.$ $\left.\chi_{\left(-1 \leq x_{2} \leq 0\right)}\left(x_{2}\right)\right]$. Then $a$ is in the classical Hardy space $H^{1}\left(\mathbb{R}^{2}\right)$. However, $T(a)$ is not in $H^{1}\left(\mathbb{R}^{2}\right)$ since $T(a) \notin L^{1}\left(\mathbb{R}^{2}\right)$. This is not surprising because the dilations play a crucial role in both singular integral operator theory and the Hardy space. Note that by the maximal characterizations of the classical and product Hardy spaces it is not difficult to see that $H^{p}\left(\mathbb{R}^{n} \times \mathbb{R}^{m}\right) \subsetneq H^{p}\left(\mathbb{R}^{n+m}\right)$, where $H^{p}\left(\mathbb{R}^{n+m}\right)$ is the classical Hardy space.

A natural question arises: is it possible that the classical singular integral operators are bounded on the product Hardy spaces? Note that the classical singular integral operators and the product Hardy spaces satisfy the different dilation properties. We would like to point out that an extension of the product singular integrals had came to light when Müller et al. [14] proved the $L^{p}, 1<p<\infty$, boundedness of Marcinkiewicz multipliers on the Heisenberg group. Nagel et al. further introduced a class of singular integrals with flag kernels on $\mathbb{R}^{n}[15]$ and recently on homogeneous groups [16].

Received by the editors August 30, 2012.

2010 Mathematics Subject Classification. Primary: 42B30; Secondary: 42B20.

Key words and phrases. Hardy Spaces, Singular Integral Operator, Discrete Calderón Reproducing Formula. 
It was pointed in [15] that singular integrals with flag kernels on $\mathbb{R}^{n} \times \mathbb{R}^{m}$ belong to the product singular integrals, see Remark 2.1.7 and Theorem 2.1.11 [15], where the characterizations in terms of the corresponding multipliers between the flag and product singular integrals are given. In this paper, we prove the analog of the one -parameter case, more precisely, that the classical Calderón-Zygmund convolution operators are bounded on the product Hardy spaces. To achieve this goal, the crucial idea is the estimate in Lemma 2.2 below, namely the classical Calderón-Zygmund convolution operators satisfy the product cancellation conditions. This fact together with the second-order difference smoothness condition on kernels carries the product almost orthogonal argument to the one-parameter case. And then applying the discrete Littlewood-Paley square function and Calderón's reproducing formula together with the Fefferman-Stein vector valued maximal function estimate gives the desired result.

In order to describe more precisely our results, we begin with recalling the product Hardy space on $\mathbb{R}^{n} \times \mathbb{R}^{m}$ and the classical Calderón-Zygmund convolution operators on $\mathbb{R}^{n+m}$. In this paper, the classical Calderón-Zygmund convolution operator (we refer reader to [18]) is defined as follows.

Definition 1.1. $\mathcal{K} \in C^{2}\left(\mathbb{R}^{n+m} \backslash\{0\}\right)$ is said to be a Calderón-Zygmund convolution kernel if there are constants $C$ and $A$ such that

$$
\left|\partial_{x}^{\alpha} \mathcal{K}(x)\right| \leq \frac{C}{|x|^{n+m+\alpha}}
$$

for $0 \leq|\alpha| \leq 2$ and $x \in \mathbb{R}^{n+m} \backslash\{0\}$, and

$$
\int_{\epsilon<|x|<N} \mathcal{K}(x) d x \leq A,
$$

whenever $0<\epsilon<N<\infty$.

We say that an operator $T$ is a classical Calderón-Zygmund convolution operator if $T f(x)=p \cdot v \cdot \mathcal{K} * f(x)$ for a Calderón-Zygmund convolution kernel $\mathcal{K}$.

It is well known that the classical Calderón-Zygmund convolution operators are bounded on $L^{p}\left(\mathbb{R}^{n+m}\right)$ for $1<p<\infty$, the classical Hardy space $H^{p}\left(\mathbb{R}^{n+m}\right)$ and from $H^{p}\left(\mathbb{R}^{n+m}\right)$ to $L^{p}\left(\mathbb{R}^{n+m}\right)$ for $p \leq 1$ but $p$ is close to 1 . See [18] for more details.

To study the boundedness of these operators on the product Hardy space, we first recall the definition of the product Hardy space $H^{p}\left(\mathbb{R}^{n} \times \mathbb{R}^{m}\right)$ (see [9] and [4] for more details).

Given $p \leq 1$ but $p$ is close to 1 and a function $\psi \in \mathcal{S}\left(\mathbb{R}^{n+m}\right)$ with the support contained in the unit ball and satisfying $\int_{\mathbb{R}^{n}} \psi\left(x_{1}, x_{2}\right) x_{1}^{\alpha} d x_{1}=\int_{\mathbb{R}^{m}} \psi\left(x_{1}, x_{2}\right) x_{2}^{\beta} d x_{2}=0$ for all $0 \leq|\alpha|,|\beta| \leq M_{p}$ where $M_{p}$ is an integer depending on $p$, and $\sum_{j, k \in \mathbb{Z}} \mid \hat{\psi}\left(2^{-j} \xi_{1}\right.$, $\left.2^{-k} \xi_{2}\right)\left.\right|^{2}=1$ for all $\xi=\left(\xi_{1}, \xi_{2}\right) \in \mathbb{R}^{n} \times \mathbb{R}^{m}$ with $\xi_{1} \neq 0$ and $\xi_{2} \neq 0$. The product Littlewood-Paley square function of $f$ is defined by

$$
g_{\psi}(f)(x)=\left\{\sum_{j, k \in \mathbb{Z}}\left|\psi_{j, k} * f(x)\right|^{2}\right\}^{\frac{1}{2}},
$$

where $\psi_{j, k}(x)=2^{j n+k m} \psi\left(2^{j} x_{1}, 2^{k} x_{2}\right)$. 
And the discrete product Littlewood-Paley square function is defined by

$$
g_{\psi}^{d}(f)(x)=\left\{\sum_{j, k \in \mathbb{Z}} \sum_{I, J}\left|\psi_{j, k} * f\left(c_{I}, c_{J}\right)\right|^{2} \chi_{I}\left(x_{1}\right) \chi_{I}\left(x_{2}\right)\right\}^{\frac{1}{2}},
$$

where $I$ and $J$ are dyadic cubes in $\mathbb{R}^{n}$ and $\mathbb{R}^{m}$ with the side lengths $l(I)=2^{-j-N}$, $l(J)=2^{-k-N}$ for some fixed large integer $N$ and the centers $c_{I}$ and $c_{J}$, respectively.

It is well known that $\left\|g_{\psi}(f)\right\|_{L^{p}\left(\mathbb{R}^{n+m}\right)} \approx\left\|g_{\psi}^{d}(f)\right\|_{L^{p}\left(\mathbb{R}^{n+m}\right)}$ and for different $\psi, \phi$ satisfying the same conditions, $\left\|g_{\psi}(f)\right\|_{L^{p}\left(\mathbb{R}^{n+m}\right)} \approx\left\|g_{\phi}(f)\right\|_{L^{p}\left(\mathbb{R}^{n+m}\right)}$. Moreover, the $H^{p}\left(\mathbb{R}^{n} \times \mathbb{R}^{m}\right)$ norm of $f$ denoted by $\|f\|_{H^{p}\left(\mathbb{R}^{n} \times \mathbb{R}^{m}\right)}$ is equivalent to $\left\|g_{\psi}^{d}(f)\right\|_{L^{p}\left(\mathbb{R}^{n+m}\right)}$. See [11] for more details.

The main results in this paper are the following

Theorem 1.2. If $T$ is a classical Caderón-Zygmund convolution operator, then $T$ is bounded on $H^{p}\left(\mathbb{R}^{n} \times \mathbb{R}^{m}\right)$ and from $H^{p}\left(\mathbb{R}^{n} \times \mathbb{R}^{m}\right)$ to $L^{p}\left(\mathbb{R}^{n+m}\right)$, for $\max \left(\frac{n}{n+1}, \frac{m}{m+1}\right)<$ $p \leq 1$.

As a consequence of Theorem 1.2, we obtain

Corollary 1.3. If $T$ is a classical Caderón-Zygmund convolution operator, then $T$ is bounded on the product $B M O\left(\mathbb{R}^{n} \times \mathbb{R}^{m}\right)$.

We remark that the range of $p$ in Theorem 1.2 can be smaller if the smoothness conditions of $\mathcal{K}$ are required to be higher. See [18] and we leave the details to the reader.

Throughout this paper, we do the following conventions:

(a) The notation $A \approx B$ means $C_{1} A \leq B \leq C_{2} A$ for some positive constants $C_{1}, C_{2}$.

(b) If $Q$ is a cube, then $c_{Q}$ is its center and $l(Q)$ is its side length.

(c) $\mathcal{Q}\left(\mathbb{R}^{n}\right)$ and $\mathcal{Q}\left(\mathbb{R}^{m}\right)$ are sets of all dyadic cubes in $\mathbb{R}^{n}$ and $\mathbb{R}^{m}$, respectively.

(d) $j \wedge j^{\prime}$ means the minimum of $j$ and $j^{\prime}$.

\section{Proof of Theorem 1.2}

The main tool in the proof of Theorem 1.2, as mentioned, is to apply the following discrete Calderón reproducing formula (See [11] for more details).

Lemma 2.1. Given $0<p \leq 1$. Suppose that $\phi(x) \in C_{0}^{\infty}\left(\mathbb{R}^{n+m}\right)$ with $\operatorname{supp}(\phi) \in\{x$ : $|x| \leq 1\}, \int_{\mathbb{R}^{n}} \phi\left(x_{1}, x_{2}\right) x_{1}^{\alpha} d x_{1}=\int_{\mathbb{R}^{m}} \phi\left(x_{1}, x_{2}\right) x_{2}^{\beta} d x_{2}=0$ for $0 \leq|\alpha|,|\beta| \leq M_{p}, M_{p}$ is a fixed large integer depending on $p$ and $\sum_{j, k \in \mathbb{Z}}\left|\hat{\phi}\left(2^{-j} \xi_{1}, 2^{-k} \xi_{2}\right)\right|^{2}=1$, for all $\xi_{1} \neq 0$ and $\xi_{2} \neq 0$. For a given $f \in L^{2}\left(\mathbb{R}^{n+m}\right) \cap H^{p}\left(\mathbb{R}^{n} \times \mathbb{R}^{m}\right)$, there exists a function $h \in L^{2}\left(\mathbb{R}^{n+m}\right) \cap H^{p}\left(\mathbb{R}^{n} \times \mathbb{R}^{m}\right)$ and a large integer $N>0$ such that $f\left(x_{1}, x_{2}\right)=$

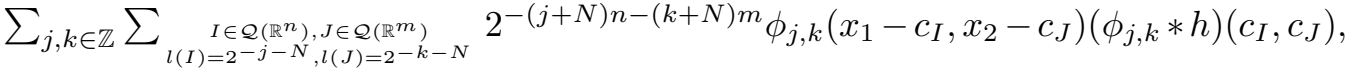
where the series converges in both $L^{2}\left(\mathbb{R}^{n+m}\right)$ and $H^{p}\left(\mathbb{R}^{n} \times \mathbb{R}^{m}\right)$. Moreover,

$$
\|f\|_{L^{2}\left(\mathbb{R}^{n+m}\right)} \approx\|h\|_{L^{2}\left(\mathbb{R}^{n+m}\right)}
$$

and

$$
\|f\|_{H^{p}\left(\mathbb{R}^{n} \times \mathbb{R}^{m}\right)} \approx\|h\|_{H^{p}\left(\mathbb{R}^{n} \times \mathbb{R}^{m}\right)} .
$$


For the proof, we refer to [11].

To prove Theorem 1.2, the crucial estimate is the following

Lemma 2.2. Suppose that $\phi(x) \in C_{0}^{\infty}\left(\mathbb{R}^{n+m}\right)$ with $\int_{\mathbb{R}^{n}} \phi\left(x_{1}, x_{2}\right) d x_{1}=\int_{\mathbb{R}^{m}}$ $\phi\left(x_{1}, x_{2}\right) d x_{2}=0$. If $\mathcal{K}$ is a Calderón-Zygmund convolution kernel given in Definition 1.1, then

$$
\left|\mathcal{K} * \phi_{j, k}(x)\right| \leq C_{\phi} \frac{2^{j n}}{1+\left|2^{j} x_{1}\right|^{n+1}} \frac{2^{k m}}{1+\left|2^{k} x_{2}\right|^{m+1}}
$$

for all $x=\left(x_{1}, x_{2}\right) \in \mathbb{R}^{n} \times \mathbb{R}^{m}$, where $C_{\phi}$ is a constant depending only on $\phi$.

Proof. Without loss of generality, we may assume that $\operatorname{supp}(\phi) \subset\{x:|x| \leq 1\}$. We prove the required estimate in four cases: (I): $\left|x_{1}\right| \geq 2^{-j+1},\left|x_{2}\right| \geq 2^{-k+1}$; (II): $\left|x_{1}\right| \geq$ $2^{-j+1},\left|x_{2}\right|<2^{-k+1}$; (III): $\left|x_{1}\right|<2^{-j+1},\left|x_{2}\right| \geq 2^{-k+1}$; (IV): $\left|x_{1}\right|<2^{-j+1},\left|x_{2}\right|<$ $2^{-k+1}$.

For case (I), by the cancellation condition on $\phi$, we have

$$
\begin{aligned}
\left|\mathcal{K} * \phi_{j, k}(x)\right|= & 2^{j n+k m} \mid \int_{\mathbb{R}^{m}} \int_{\mathbb{R}^{n}}\left\{\left(\mathcal{K}\left(x_{1}-y_{1}, x_{2}-y_{2}\right)-\mathcal{K}\left(x_{1}, x_{2}-y_{2}\right)\right)\right. \\
& \left.-\left(\mathcal{K}\left(x_{1}-y_{1}, x_{2}\right)-\mathcal{K}\left(x_{1}, x_{2}\right)\right)\right\} \phi\left(2^{j} y_{1}, 2^{k} y_{2}\right) d y_{1} d y_{2} \mid .
\end{aligned}
$$

Note that $\left(\mathcal{K}\left(x_{1}-y_{1}, x_{2}-y_{2}\right)-\mathcal{K}\left(x_{1}, x_{2}-y_{2}\right)\right)-\left(\mathcal{K}\left(x_{1}-y_{1}, x_{2}\right)-\mathcal{K}\left(x_{1}, x_{2}\right)\right)=$ $\int_{0}^{1} \int_{0}^{1} \partial_{s}^{1} \partial_{t}^{1}\left[\mathcal{K}\left(x_{1}-s y_{1}, x_{2}-t y_{2}\right)\right] d s d t=\int_{0}^{1} \int_{0}^{1} \sum_{i=1}^{n} \sum_{j=1}^{m} y_{1 i} y_{2 j} \partial_{x_{1 i}}^{1} \partial_{x_{2 j}}^{1} K\left(x_{1}-s y_{1}\right.$, $\left.\left.x_{2}-t y_{2}\right)\right] d s d t$, where $y_{1}=\left(y_{11}, y_{12}, \ldots, y_{1 n}\right), y_{2}=\left(y_{21}, y_{22}, \ldots, y_{2 m}\right)$. Applying the hypothesis on $\mathcal{K}$, that is, the second-order difference smoothness condition, yields

$$
\begin{aligned}
\left|\mathcal{K} * \phi_{j, k}(x)\right| & \leq C 2^{j n+k m} \int_{\mathbb{R}^{m}} \int_{\mathbb{R}^{n}} \frac{\left|y_{1}\right|\left|y_{2}\right|}{\left(\left|x_{1}\right|+\left|x_{2}\right|\right)^{m+n+2}}\left|\phi\left(2^{j} y_{1}, 2^{k} y_{2}\right)\right| d y_{1} d y_{2} \\
& \leq C_{\phi} \frac{2^{j n}}{1+\left|2^{j} x_{1}\right|^{n+1}} \frac{2 k m}{1+\left|2^{k} x_{2}\right|^{m+1}} .
\end{aligned}
$$

For case (II), by the hypothesis that $\int_{\mathbb{R}^{n}} \phi\left(x_{1}, x_{2}\right) d x_{1}=0$, we write

$$
\begin{aligned}
\left|\mathcal{K} * \phi_{j, k}(x)\right|= & 2^{j n+k m} \mid \int_{\mathbb{R}^{m}} \int_{\mathbb{R}^{n}}\left(\mathcal{K}\left(x_{1}-y_{1}, x_{2}-y_{2}\right)\right. \\
& \left.-\mathcal{K}\left(x_{1}, x_{2}-y_{2}\right)\right) \phi\left(2^{j} y_{1}, 2^{k} y_{2}\right) d y_{1} d y_{2} \mid
\end{aligned}
$$

Applying the mean value theorem and the hypothesis on $\mathcal{K}$ implies

$$
\begin{aligned}
\left|\mathcal{K} * \phi_{j, k}(x)\right| & \leq C 2^{j n+k m} \int_{\left|y_{2}\right| \leq 2^{-k}} \int_{\left|y_{1}\right| \leq 2^{-j}} \frac{\left|y_{1}\right|}{\left(\left|x_{1}\right|+\left|x_{2}-y_{2}\right|\right)^{n+m+1}} d y_{2} d y_{1} \\
& \leq C_{\phi} 2^{k m} \frac{2^{j n}}{1+\left|2^{j} x_{1}\right|^{n+1}} \leq C_{\phi} \frac{2^{j n}}{1+\left|2^{j} x_{1}\right|^{n+1}} \frac{2^{k m}}{1+\left|2^{k} x_{2}\right|^{m+1}} .
\end{aligned}
$$

Since the cases (II) and (III) are symmetric, so the proof for case (III) follows from the proof for case (II). 
For the last case (IV), let $\eta_{1} \in C_{0}^{\infty}\left(\mathbb{R}^{n}\right)$ with $0 \leq \eta_{1}\left(x_{1}\right) \leq 1$ and $\eta_{1}\left(x_{1}\right)=1$ when $\left|x_{1}\right| \leq 4$, and $\eta_{1}\left(x_{1}\right)=0$ when $\left|x_{1}\right| \geq 8$. Set $\eta_{2}\left(x_{2}\right)$ similarly. Then

$$
\begin{aligned}
& \left|\mathcal{K} * \phi_{j, k}(x)\right| \\
& =2^{j n+k m}\left|\int_{\mathbb{R}^{m}} \int_{\mathbb{R}^{n}} \mathcal{K}\left(y_{1}, y_{2}\right) \phi\left(2^{j}\left(x_{1}-y_{1}\right), 2^{k}\left(x_{2}-y_{2}\right)\right) \eta_{1}\left(2^{j} y_{1}\right) \eta_{2}\left(2^{k} y_{2}\right) d y_{1} d y_{2}\right| \\
& \leq 2^{j n+k m} \mid \int_{\mathbb{R}^{m}} \int_{\mathbb{R}^{n}} \mathcal{K}\left(y_{1}, y_{2}\right)\left(\phi\left(2^{j}\left(x_{1}-y_{1}\right), 2^{k}\left(x_{2}-y_{2}\right)\right)\right. \\
& \left.\quad-\phi\left(2^{j} x_{1}, 2^{k} x_{2}\right)\right) \eta_{1}\left(2^{j} y_{1}\right) \eta_{2}\left(2^{k} y_{2}\right) d y_{1} d y_{2} \mid \\
& \left.+2^{j n+k m} \mid \int_{\mathbb{R}^{m}} \int_{\mathbb{R}^{n}} \mathcal{K}\left(y_{1}, y_{2}\right) \eta_{1}\left(2^{j} y_{1}\right) \eta_{2}\left(2^{k} y_{2}\right) \phi\left(2^{j} x_{1}, 2^{k} x_{2}\right)\right) d y_{1} d y_{2} \mid .
\end{aligned}
$$

Using the size condition on $\mathcal{K}$ and smoothness condition on $\phi$ for the first term and the fact that $\hat{\mathcal{K}}$ is bounded for the second term above gives

$$
\begin{array}{rl}
\mid \mathcal{K} & * \phi_{j, k}(x) \mid \\
\leq & C_{\phi} 2^{j n+k m} \int_{\left|y_{2}\right| \leq 2^{-k+3}} \int_{\left|y_{1}\right| \leq 2^{-j+3}} \frac{1}{\left(\left|y_{1}\right|+\left|y_{2}\right|\right)^{n+m}}\left(\left|2^{j} y_{1}\right|+\left|2^{k} y_{2}\right|\right) d y_{1} d y_{2} \\
& +C_{\phi}\left|\int_{\mathbb{R}^{m}} \int_{\mathbb{R}^{n}} \hat{\mathcal{K}}\left(\xi_{1}, \xi_{2}\right) \hat{\eta}_{1}\left(2^{-j} \xi_{1}\right) \hat{\eta}_{2}\left(2^{-k} \xi_{2}\right) d \xi_{1} d \xi_{2}\right| \\
\leq & C_{\phi} 2^{j n+k m} \leq C_{\phi} \frac{2^{j n}}{1+\left|2^{j} x_{1}\right|^{n+1}} \frac{2^{k m}}{1+\left|2^{k} x_{2}\right|^{m+1}} .
\end{array}
$$

The proof of Lemma 2.2 is concluded.

We remark that it is a well-known estimate in Lemma 2.2 if $\mathcal{K}$ is a product singular integral kernel, see [8]. As mentioned in section 1, however, the above proof indeed implies that if $\mathcal{K}$ is a classical Calderón-Zygmund convolution kernel then

$$
\left|\int_{\mathbb{R}^{m}} \int_{\mathbb{R}^{n}} \mathcal{K}\left(y_{1}, y_{2}\right) \eta_{1}\left(2^{j} y_{1}\right) \eta_{2}\left(2^{k} y_{2}\right) d y_{1} d y_{2}\right| \leq C
$$

uniformly for all bump functions $\eta_{1}, \eta_{2}$ and $j, k$. This crucial estimate means that classical Calderón-Zygmund convolution kernels $\mathcal{K}$ satisfy the product cancellation conditions.

As a consequence of Lemma 2.2, we get the following product almost orthogonal estimates for the classical Calderón-Zygmund convolution kernels.

Lemma 2.3. Under the hypothesis of Lemma 2.2,

$$
\left|\phi_{j, k} * \mathcal{K} * \phi_{j^{\prime}, k^{\prime}}(x)\right| \leq C_{\phi} 2^{-\left|j-j^{\prime}\right|} 2^{-\left|k-k^{\prime}\right|} \frac{2^{\left(j \wedge j^{\prime}\right) n}}{1+\left|2^{j \wedge j^{\prime}} x_{1}\right|^{n+1}} \frac{2^{\left(k \wedge k^{\prime}\right)^{m}}}{1+\left|2^{k \wedge k^{\prime}} x_{2}\right|^{m+1}}
$$

for all $x=\left(x_{1}, x_{2}\right) \in \mathbb{R}^{n} \times \mathbb{R}^{m}$.

The proof of the Lemma 2.3 is based on the following two observations: (1) convolution operation is commutative, i.e., $\phi_{j, k} * \mathcal{K} * \phi_{j^{\prime}, k^{\prime}}(x)=\mathcal{K} *\left(\phi_{j, k} *\right.$ $\left.\phi_{j^{\prime}, k^{\prime}}\right)(x) ;(2) \phi_{j, k} * \phi_{j^{\prime}, k^{\prime}}$ satisfies the same estimates as $\phi_{j \wedge j^{\prime}, k \wedge k^{\prime}}$ with the bound $C 2^{-\left|j-j^{\prime}\right|} 2^{-\left|k-k^{\prime}\right|}$. The detail is left to readers. 
To prove Theorem 1.2, we also need the following strong maximal function estimates:

Lemma 2.4. Suppose that $\max \left(\frac{n}{n+1}, \frac{m}{m+1}\right)<\delta \leq 1, F \in L^{2}\left(\mathbb{R}^{n+m}\right)$ and $N$ is an integer. If $I^{\prime} \in \mathcal{Q}\left(\mathbb{R}^{n}\right), J^{\prime} \in \mathcal{Q}\left(\mathbb{R}^{m}\right)$ with $l\left(I^{\prime}\right)=2^{-j^{\prime}}, l\left(J^{\prime}\right)=2^{-k^{\prime}}$, respectively. Then for any $u=\left(u_{1}, u_{2}\right), v=\left(v_{1}, v_{2}\right) \in I^{\prime} \times J^{\prime}$,

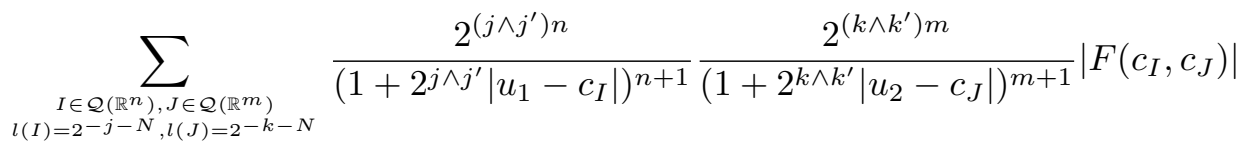

$$
\begin{aligned}
& \leq C 2^{n\left(j \wedge j^{\prime}\right)(1-1 / \delta)+n j / \delta} 2^{m\left(k \wedge k^{\prime}\right)(1-1 / \delta)+m k / \delta} \\
& \times\left\{M_{S}\left[\left(\sum_{\substack{\left.I \in \mathcal{Q}_{(\mathbb{R}}^{n}\right), J \in \mathcal{Q}\left(\mathbb{R}^{m}\right) \\
l(I)=2^{-j-N}, l(J)=2^{-k-N}}}\left|F\left(c_{I}, c_{J}\right)\right|^{2} \chi_{I} \chi_{J}\right)^{\delta / 2}\right]\right\}^{1 / \delta}
\end{aligned}
$$

where $M_{s}$ is the strong maximal function.

For the proof, we refer readers to [5].

Proof of Theorem 1.2. Since $L^{2}\left(\mathbb{R}^{n+m}\right) \cap H^{p}\left(\mathbb{R}^{n} \times \mathbb{R}^{m}\right)$ is dense in $H^{p}\left(\mathbb{R}^{n} \times \mathbb{R}^{m}\right)$, we just need to show that

$$
\|T f\|_{H^{p}\left(\mathbb{R}^{n} \times \mathbb{R}^{m}\right)} \leq C\|f\|_{H^{p}\left(\mathbb{R}^{n} \times \mathbb{R}^{m}\right)}
$$

for all $f \in L^{2}\left(\mathbb{R}^{n+m}\right) \cap H^{p}\left(\mathbb{R}^{n} \times \mathbb{R}^{m}\right)$.

Let $I^{\prime} \in \mathcal{Q}\left(\mathbb{R}^{n}\right), J^{\prime} \in \mathcal{Q}\left(\mathbb{R}^{m}\right)$ with $l\left(I^{\prime}\right)=2^{-j^{\prime}}, l\left(J^{\prime}\right)=2^{-k^{\prime}}$, respectively. By the discrete product Littlewood-Paley square function we write

$$
\left|g_{\psi}^{d}(T f)(x)\right|^{2}=\sum_{j^{\prime}, k^{\prime} \in \mathbb{Z}} \sum_{\substack{I^{\prime} \in \mathcal{Q}\left(\mathbb{R}^{n}\right), J^{\prime} \in \mathcal{Q}\left(\mathbb{R}^{m}\right) \\ l\left(I^{\prime}\right)=2^{-j^{\prime}}, l\left(J^{\prime}\right)=2^{-k^{\prime}}}}\left|\psi_{j^{\prime}, k^{\prime}} * \mathcal{K} * f\left(c_{I^{\prime}}, c_{J^{\prime}}\right)\right|^{2} \chi_{I^{\prime}}\left(x_{1}\right) \chi_{J^{\prime}}\left(x_{2}\right) .
$$

For any $u=\left(u_{1}, u_{2}\right), x=\left(x_{1}, x_{2}\right) \in I^{\prime} \times J^{\prime}$, applying Lemma 2.1 gives

$$
\begin{aligned}
\left|\psi_{j^{\prime}, k^{\prime}} * \mathcal{K} * f(u)\right|= & \mid \sum_{j, k \in \mathbb{Z}} \sum_{\substack{I \in \mathcal{Q}\left(\mathbb{R}^{n}\right), J \in \mathcal{Q}\left(\mathbb{R}^{m}\right) \\
l(I)=2-j-N, l(J)=2^{-k-N}}} 2^{-(j+N) n-(k+N) m} \psi_{j^{\prime}, k^{\prime}} * \mathcal{K} \\
& * \psi_{j, k}\left(u_{1}-c_{I}, u_{2}-c_{J}\right)\left(\psi_{j, k} * h\right)\left(c_{I}, c_{J}\right) \mid .
\end{aligned}
$$

By Lemma 2.3, we have

$$
\begin{aligned}
& \left|\psi_{j^{\prime}, k^{\prime}} * \mathcal{K} * f(u)\right| \\
& \leq C \sum_{j, k \in \mathbb{Z}} \sum_{\substack{I \in \mathcal{Q}\left(\mathbb{R}^{n}\right), J \in \mathcal{Q}\left(\mathbb{R}^{m}\right) \\
l(I)=2^{-j-N}, l(J)=2^{-k-N}}} 2^{-j n-k m} 2^{-\left|j-j^{\prime}\right|} 2^{-\left|k-k^{\prime}\right|} \\
& \frac{2^{\left(j \wedge j^{\prime}\right) n}}{1+\left|2^{j \wedge j^{\prime}}\left(u_{1}-c_{I}\right)\right|^{n+1}} \frac{2^{\left(k \wedge k^{\prime}\right) m}}{1+\left|2^{k \wedge k^{\prime}}\left(u_{2}-c_{J}\right)\right|^{m+1}}\left|\psi_{j, k} * h\right|\left(c_{I}, c_{J}\right) .
\end{aligned}
$$


Applying Lemma 2.4 with $F=\psi_{j, k} * h$ and $\max \left(\frac{n}{n+1}, \frac{m}{m+1}\right)<\delta<p$ implies

$$
\begin{aligned}
& \left|\psi_{j^{\prime}, k^{\prime}} * \mathcal{K} * f(u)\right| \\
& \leq C \sum_{j, k \in \mathbb{Z}} 2^{-j n-k m} 2^{-\left|j-j^{\prime}\right|} 2^{-\left|k-k^{\prime}\right|} 2^{n\left(j \wedge j^{\prime}\right)(1-1 / \delta)+n j / \delta} 2^{m\left(k \wedge k^{\prime}\right)(1-1 / \delta)+m k / \delta}
\end{aligned}
$$

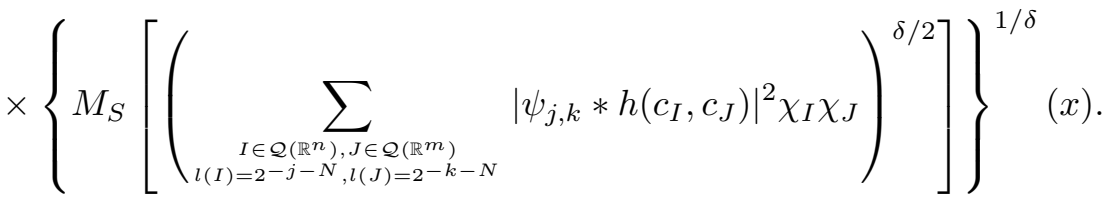

Therefore,

$$
\begin{array}{rl}
\left|g_{\psi}^{d}(T f)(x)\right|^{2} & C \sum_{j^{\prime}, k^{\prime} \in \mathbb{Z}} \sum_{\substack{I^{\prime} \in \mathcal{Q}\left(\mathbb{R}^{n}\right), J^{\prime} \in \mathcal{Q}\left(\mathbb{R}^{m}\right) \\
l\left(I^{\prime}\right)=2-j^{\prime}, l\left(J^{\prime}\right)=2^{-k^{\prime}}}} \\
& {\left[\sum_{j, k \in \mathbb{Z}} 2^{-j n-m k} 2^{-\left|j-j^{\prime}\right|} 2^{-\left|k-k^{\prime}\right|} 2^{n\left(j \wedge j^{\prime}\right)(1-1 / \delta)+n j / \delta} 2^{m\left(k \wedge k^{\prime}\right)(1-1 / \delta)+m k / \delta}\right.} \\
& \left\{M_{S}\left[\left(\begin{array}{c}
\substack{I \in \mathcal{Q}\left(\mathbb{R}^{n}\right), J \in \mathcal{Q}\left(\mathbb{R}^{m}\right) \\
l(I)=2-j-N, l(J)=2-k-N \\
M^{\prime}\left(x_{2}\right) .} \\
\chi_{I^{\prime}}\left(x_{1}\right) \chi_{J^{\prime}}\left(\left.\psi_{j, k} * h\left(c_{I}, c_{J}\right)\right|^{2} \chi_{I} \chi_{J}\right.
\end{array}\right)^{1 / \delta}\right\}^{2}(x)\right]^{2}
\end{array}
$$

Summing up $I^{\prime}, J^{\prime}$ and applying the Cauchy-Schwartz inequality, we get

$$
\begin{aligned}
& \left|g_{\psi}^{d}(T f)(x)\right|^{2} \\
& \leq C \sum_{j^{\prime}, k^{\prime} \in \mathbb{Z}}\left(\sum_{j, k \in \mathbb{Z}} 2^{-n j-m k} 2^{-\left|j-j^{\prime}\right|} 2^{-\left|k-k^{\prime}\right|} 2^{n\left(j \wedge j^{\prime}\right)(1-1 / \delta)+n j / \delta} 2^{m\left(k \wedge k^{\prime}\right)(1-1 / \delta)+m k / \delta}\right) \\
& \left(\sum_{j, k \in \mathbb{Z}} 2^{-n j-m k} 2^{-\left|j-j^{\prime}\right|} 2^{-\left|k-k^{\prime}\right|} 2^{n\left(j \wedge j^{\prime}\right)(1-1 / \delta)+n j / \delta} 2^{m\left(k \wedge k^{\prime}\right)(1-1 / \delta)+m k / \delta}\right. \\
& \left.\left\{M_{S}\left[\left(\sum_{\substack{I \in \mathcal{Q}\left(\mathbb{R}^{n}\right), J \in \mathcal{Q}\left(\mathbb{R}^{m}\right) \\
l(I)=2^{-j-N}, l(J)=2^{-k-N}}}\left|\psi_{j, k} * h\left(c_{I}, c_{J}\right)\right|^{2} \chi_{I} \chi_{J}\right)^{\delta / 2}\right]\right\}^{2 / \delta}(x)\right)
\end{aligned}
$$




$$
\begin{aligned}
& \leq C \sum_{j^{\prime}, k^{\prime} \in \mathbb{Z}} \sum_{j, k \in \mathbb{Z}} 2^{-n j-m k} 2^{-\left|j-j^{\prime}\right|} 2^{-\left|k-k^{\prime}\right|} 2^{n\left(j \wedge j^{\prime}\right)(1-1 / \delta)+n j / \delta} 2^{m\left(k \wedge k^{\prime}\right)(1-1 / \delta)+m k / \delta}
\end{aligned}
$$

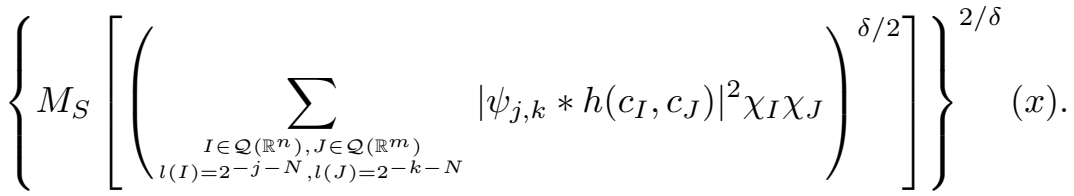

Changing the order of the series implies

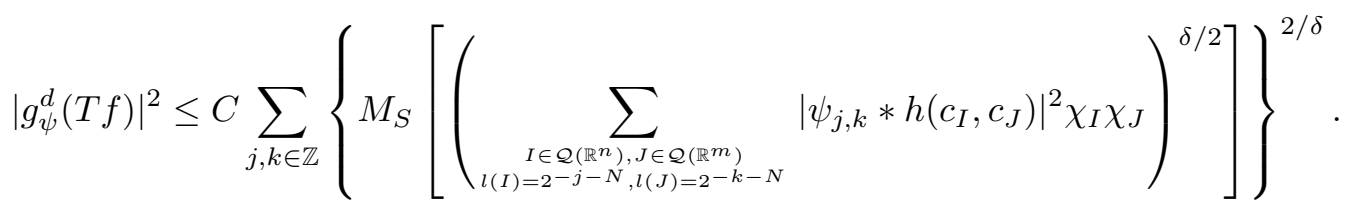

Applying the Fefferman-Stein vector-valued strong maximal inequality (see [7] and [18] for more details) on $L^{p / \delta}\left(\ell^{2 / \delta}\right)$ yields

$$
\begin{aligned}
& \|T(f)\|_{H^{p}\left(\mathbb{R}^{n} \times \mathbb{R}^{m}\right)}=\left\|g_{\psi}^{d}(T f)\right\|_{L^{p}\left(\mathbb{R}^{n+m}\right)}
\end{aligned}
$$

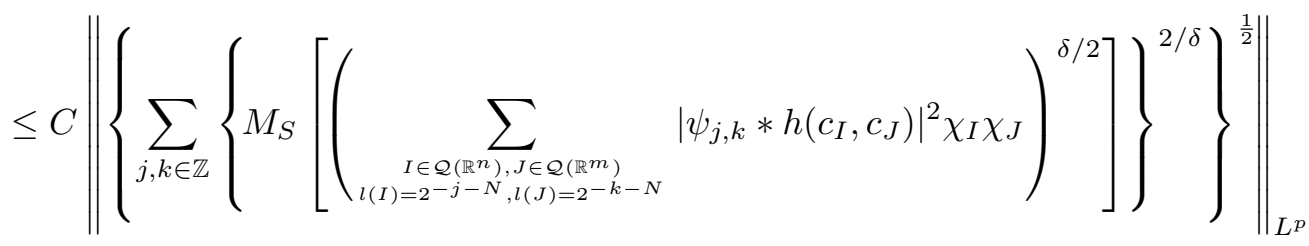

$$
\begin{aligned}
& \leq C\left\|\left\{\sum_{j, k \in \mathbb{Z}} \sum_{\substack{I \in \mathcal{Q}\left(\mathbb{R}^{n}\right), J \in \mathcal{Q}\left(\mathbb{R}^{m}\right) \\
l(I)=2^{-j-N}, l(J)=2^{-k-N}}}\left|\psi_{j, k} * h\left(c_{I}, c_{J}\right)\right|^{2} \chi_{I}\left(x_{1}\right) \chi_{J}\left(x_{2}\right)\right\}^{\frac{1}{2}}\right\|_{L^{p}} \\
& =C\|h\|_{H^{p}\left(\mathbb{R}^{n} \times \mathbb{R}^{m}\right)} \leq C\|f\|_{H^{p}\left(\mathbb{R}^{n} \times \mathbb{R}^{m}\right)} .
\end{aligned}
$$

By a general result, that is, if an operator is bounded on both $L^{2}$ and $H^{p}$, then $T$ extends to a bounded operator from $H^{p}$ to $L^{p}$. Here $H^{p}$ are both the classical and product Hardy spaces. See [12] for more details. The proof of Theorem 1.2 is concluded.

By the duality, Corollary 1.3 follows directly from Theorem 1.2.

\section{Acknowledgments}

The author wish to express his sincere appreciation to the referee for his/her very helpful comments. The author would like to thank Elias Stein for his very constructive suggestions. Finally, the author would also like to thank Yongsheng Han for his great support. The author is supported by SRF for the Doctoral Program of Higher Education (Grant No. 20104402120002).

\section{References}

[1] L. Carleson, A counterexample for measures bounded on Hp for the bi-disc, Mittag Leffler Report No. 7, 1974.

[2] S.Y. Chang and R. Fefferman, A continuous version of duality of $H^{1}$ with BMO on the bidisc, Ann. Math. 112(1) (1980), 179-201.

[3] S.Y. Chang and R. Fefferman, Some recent developments in Fourier analysis and $H^{p}$-theory on product domains, Bull. Amer. Math. Soc. (N.S.) 12(1) (1985), 1-43. 
[4] R. Fefferman, Calderón-Zygmund theory for product domains: Hp spaces, Proc. Natl. Acad. Sci. USA 83(4), (1986), 840-843.

[5] M. Frazier and B. Jawerth, A discrete transform and decompositions of distribution spaces, J. Funct. Anal. 93(1), (1990), 34-170.

[6] C. Fefferman and E.M. Stein, Hardy spaces of several variables, Acta Math. 129 (1972), 137-193.

[7] C. Fefferman and E.M. Stein, Some maximal inequalities, Amer. J. Math. 93 (1971), 107-115.

[8] R. Fefferman and E.M. Stein, Singular integrals on product spaces, Adv. Math. 45 (1982), $117-143$.

[9] R. Gundy and E.M. Stein, Hp theory for the poly-disc, Proc. Natl. Acad. Sci. USA 76 (1979), 1026-1029.

[10] Y.S. Han, M.Y. Lee, C.C. Lin and Y.C. Lin, Calderón-Zygmund operators on product Hardy spaces, J. Funct. Anal. 258 (2010), 2834-2861.

[11] Y.S. Han, G.Z. Lu and K. Zhao, Discrete Calderón's identity, atomic decomposition and boundedness criterion of operators on multiparameter hardy spaces, J. Geom. Anal. 20 (2010), 670-689.

[12] Y.S. Han, J. Li, G.Z. Lu and P.Y. Wang, $H^{p} \rightarrow H^{p}$ boundedness implies $H^{p} \rightarrow L^{p}$ boundedness, Forum Math. 23 (2011), 729-756.

[13] J.L. Journé, Calderón-Zygmund operators on product spaces, Rev. Mat. Iberoamericana 1 (1985), 55-91.

[14] D. Müller, F. Ricci and E. Stein, Marcinkiewicz multipliers and multi-parameter structure on Heisenberg(-type) groups, I, Invent. Math. 119 (1995), 119-233.

[15] A. Nagel, F. Ricci and E. Stein, Singular integrals with flag kernels and analysis on quadratic CR manifolds, J. Funct. Anal. 181 (2001), 29-118.

[16] A. Nagel, F. Ricci, E. Stein and S. Wainger, Singular integrals with flag kernels on homogeneous groups: I, Rev. Mat. Iberoamericana 28 (2012), 631-722.

[17] E.M. Stein and G. Weiss, On the theory of harmonic functions of several variables. I. The theory of $H$ p-spaces, Acta Math. 103 (1960), 25-62.

[18] E.M. Stein, Harmonic analysis: real variable methods, orthogonality and oscillatory integrals, Princeton University Press, Princeton, NJ, 1993.

Department of mathematics, Shantou University, Shantou city, Guangdong Province 515063, People's Republic of China

E-mail address: cqtan@stu.edu.cn 
